\title{
Trivium
}

Revue franco-allemande de sciences humaines et sociales - Deutsch-französische Zeitschrift für Geistesund Sozialwissenschaften

$7 \mid 2010$

Max Weber et la bureaucratie

\section{L'histoire de l'État comme histoire de la bureaucratie}

\section{Andreas Anter}

Traducteur : Olivier Mannoni

\section{(2) OpenEdition}

Journals

Édition électronique

URL : http://journals.openedition.org/trivium/3794

DOI : $10.4000 /$ trivium.3794

ISSN : 1963-1820

Éditeur

Les éditions de la Maison des sciences de l'Homme

Référence électronique

Andreas Anter, «L'histoire de l'État comme histoire de la bureaucratie », Trivium [En ligne], 7 | 2010, mis en ligne le 06 décembre 2010, consulté le 10 décembre 2020. URL : http://journals.openedition.org/ trivium/3794; DOl : https://doi.org/10.4000/trivium.3794

Ce document a été généré automatiquement le 10 décembre 2020.

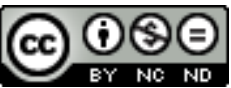

Les contenus des la revue Trivium sont mis à disposition selon les termes de la Licence Creative Commons Attribution - Pas d'Utilisation Commerciale - Pas de Modification 4.0 International. 


\title{
L'histoire de l'État comme histoire de la bureaucratie
}

\author{
Andreas Anter
}

Traduction : Olivier Mannoni

\section{NOTE DE L'ÉDITEUR}

Nous remercions Andreas Anter et la maison d'édition Duncker \& Humblot de nous avoir accordé l'autorisation de traduire ce texte dans le présent numéro.

\section{L'histoire de l'État comme histoire de la bureaucratie}

« Les fonctionnaires sont des gens très capables, mais dans une seule spécialité ; quand une question est de leur ressort, il leur suffit d'un mot pour saisir toute une série de pensées, mais s'il s'agit d'une chose qui sort de leur rayon, on peut passer des heures à la leur expliquer, ils remuent la tête poliment mais ils ne comprennent pas un

mot».

Franz Kafka, Le Château ${ }^{1}$

Max Weber s'intéresse moins à la genèse de l' «État » qu'à sa forme structurelle et à son mode de fonctionnement spécifiques : la bureaucratie. On le voit presque dans tous les contextes où il considère l'État sous des points de vue historiques. Lorsqu'il voit surgir partout les «germes" du système étatique moderne «en parallèle avec le développement des entités bureaucratiques $»^{2}$ et considère la bureaucratie comme «la spore de l'État occidental moderne ${ }^{3}$, l'histoire de l'État moderne devient pour lui l'histoire de la bureaucratie moderne. Comme celle-ci n'est pas au premier plan dans la seule perspective historique, mais aussi dans l'analyse de l'État de son époque, on peut 
lire la théorie de l'État de Weber comme une théorie de la bureaucratie et sa théorie de la bureaucratie comme une théorie de l'État.

2 La théorie de la bureaucratie est certes l'un des thèmes de son œuvre les plus fréquemment étudiés, mais on ne l'a pas encore abordée à ce jour sous l'angle de sa dimension de théorie de l'État. Cela peut tenir au fait que la conception qui préside à cette théorie paraît aussi impressionnante que cohérente. Notre but, dans les pages qui suivent, ne sera pas seulement de faire ressortir le lien entre la théorie de l'État et la théorie de la bureaucratie, mais aussi de montrer que et comment, sur des points décisifs, Max Weber renoue avec des positions de la théorie de la bureaucratie au XIX ${ }^{\mathrm{e}}$ et au début du XX $\mathrm{XX}^{\mathrm{e}}$ siècles, les modifie et les développe. Son modèle de bureaucratie, qui a été suffisamment étudié, loué et critiqué ${ }^{4}$, ne nous intéressera ici qu'en ce qui concerne son lien avec les aspects relevant de la théorie de l'État.

3 S'appuyant sur de très nombreux exemples, Max Weber démontre et diagnostique le lien entre la formation des États et la bureaucratisation en Occident. "L'État moderne ", écrit-il laconiquement, "naît lorsque le prince prend sous son égide » la monopolisation de la violence et la bureaucratisation de l'administration ${ }^{5}$. Les "puissances étatiques continentales» du début des temps modernes se sont " entièrement concentrées entre les mains des princes qui ont pris de la manière la plus résolue le chemin de la bureaucratisation de l'administration $»^{6}$. Se fondant sur ses considérations historiques, il conclut que "plus le temps passe, plus le grand État moderne a tout simplement besoin d'une base bureaucratique ». Pour Max Weber, la cause première tient aux "demandes croissantes adressées à l'administration", notamment en raison des missions de politique sociale que l'on fait pour partie «endosser» à l'État, et que celui-ci usurpe pour l'autre partie ${ }^{7}$. Il prend ainsi une position sur laquelle règne aujourd'hui un consensus dans les textes de politologie et de sciences juridiques. Que l'extension des fonctions de l'État en général, et en particulier la politique sociale et le système de l'État-providence, aient contribué de manière décisive à la bureaucratisation, est une idée que nul ne conteste dans la recherche récente ${ }^{8}$.

Weber n'est d'ailleurs pas le premier à faire ce constat. Robert von Mohl considère déjà que l'extension des missions de l'État constitue le facteur décisif de la bureaucratisation et constate que « de nouvelles exigences et de nouveaux besoins » élargissent le champ de l'activité de l'État et augmentent du même coup le volume de l'appareil des fonctionnaires. Josef Olszewski en conclut qu'avec la naissance des grands États modernes apparaît "comme conséquence inéluctable» la «centralisation de l'administration" et la "polycratie » bureaucratique ${ }^{10}$, tandis que la "bureaucratie d'État» est "renforcée jusqu'à atteindre la plénitude suprême de ses pouvoirs ${ }^{11}$. Weber voit bien que le système de l'État social qui commence à se constituer à son époque ne peut qu'accélérer le processus de la bureaucratisation. On a donc du mal à comprendre comment on peut affirmer qu'il n'a " pas vu » l'État social qui s'annonçait alors ${ }^{12}$. Tout au contraire, il observe très précisément l'État de son temps, qui devient à la fin du XIX siècle un "prestataire » et développe un degré de " prévoyance ${ }^{13}$ que d'autres États européens n'atteindront que beaucoup plus tard. Ce progrès suppose inévitablement une contrepartie. L'extension quantitative et qualitative des missions de l'État débouche nécessairement sur une expansion et une différenciation, une formalisation et une professionnalisation de l'appareil administratif: une «bureaucratisation de la bureaucratie ${ }^{14}$. 
5 Dans la liste considérable des autres facteurs qui ont contribué à la bureaucratisation, on trouve la création d'armées permanentes, le développement de l'économie monétaire, un système fiscal rationnel et une technique avancée de transports et de communications. Weber apporte la preuve d'une symbiose parfaite entre l'État, la technique et la bureaucratie. Il ne considère pas le chemin de fer et le télégraphe uniquement comme des "moteurs de la bureaucratisation", mais aussi comme des instruments de domination de l'État moderne, lequel ne peut selon lui être administré que « parce qu'il est le maître du réseau télégraphique, et que la poste et les chemins de fer sont à sa disposition $»^{15}$. Il cite quantité de facteurs qui ont contribué à la bureaucratisation et qui interagissent les uns avec les autres. Les questions de causalité ne l'intéressent certes pas, mais on voit clairement que deux facteurs occupent une place prédominante: les lois internes qui régissent la sphère administrative et le développement économique. D'une part, l'État comme institution rationnelle, avec sa sécurité juridique et sa calculabilité, promeut de manière décisive le capitalisme mieux: en permet seul l'existence - et d'autre part la forme structurelle et le fonctionnement de l'État s'adaptent de manière presque mimétique à celles de l'économie capitaliste. L'entreprise capitaliste moderne a besoin "pour exister d'une justice et d'une administration dont le fonctionnement [...] peut être calculé rationnellement, de la même manière que l'on calcule le rendement prévisible d'une machine ", tandis que l'État moderne devient lui-même une "entreprise » organisée " comme une usine ${ }^{16}$.

6 Max Weber fait naître l'image d'une analogie parfaite qu'il ne se lasse pas de décrire. « De la même manière que ce qu'on appelle le progrès vers le capitalisme est, depuis le Moyen Âge, le critère sans équivoque de la modernisation de l'économie, l'évolution vers la fonction publique [...] bureaucratique est le critère tout aussi clair de la modernisation de l'État ${ }^{17}$ ». Il établit le diagnostic d'une "parenté sociologique » entre le capitalisme et la bureaucrati $e^{18}$, qui se sont matérialisés et encouragés au cours d'un processus d'interaction très réussi ayant débouché sur leur victoire commune. Ses considérations sur la naissance de la bureaucratie, sous l'angle de la sociologie de la domination, ne peuvent et ne veulent certes pas remplacer une histoire de l'État moderne, mais fournissent bon nombre de positions théoriques et historiques qui peuvent et doivent servir de base à une étude historique. Elles montrent notamment que toute histoire de l'État moderne doit nécessairement intégrer ces deux dimensions qui, plus que toute autre, ont forgé l'État : le capitalisme et la bureaucratie.

7 En ce qui concerne le phénomène historique et actuel de l'État institutionnel moderne et rationnel, Weber s'intéresse particulièrement à la catégorie qui forme «le pilier de l'État moderne $»^{19}$ : les fonctionnaires spécialisés apparaissent, dans son œuvre, comme les protagonistes décisifs de la genèse de la forme étatique, de telle sorte que «toute l'histoire de l'évolution de l'État moderne » est pour lui «identique à l'histoire de la fonction publique moderne $»^{20}$. Cette découverte, qui fait aujourd'hui partie «de la culture générale en Europe centrale», comme l'affirme avec optimisme Otto Kimminich ${ }^{21}$, mais se trouve au moins «dans presque tous les manuels de droit public » ${ }^{22}$, n'est pas révolutionnaire, mais s'inscrit déjà dans le patrimoine de connaissances établi au sein de la littérature scientifique de son époque ${ }^{23}$. Max Weber veut souligner une constellation historique singulière lorsqu'il dit qu' « aucun pays et aucune époque n'a connu, dans le sens où le connaît l'Occident moderne ", « le caractère absolument et inéluctablement captif de toute notre vie, des conditions fondamentales politiques, 
techniques et économiques de notre existence, dans l'armature d'une organisation de fonctionnaires ayant reçu une formation spécialisée $»^{24}$. Cette ligne existentielle majeure, associée au topos wébérien du Gehäuse [la «cage» ou "l'habitat» (N.d.T.)], régit son observation et son évaluation de la fonction publique, qu'il ne considère pas du tout comme un simple instrument, mais aussi comme un vecteur de la domination de l'État. On le voit sans doute de la manière la plus claire dans son texte " Parlement et gouvernement dans l'Allemagne réorganisée ", qui constitue, à en croire son sous-titre, une "critique de la fonction publique». Il y déplore le fait que des fonctionnaires prennent des décisions «sur tous nos besoins quotidiens et nos doléances quotidiennes", et que "la véritable domination" dans l'État moderne se trouve «nécessairement et inévitablement entre les mains du corps des fonctionnaires " ${ }^{25}$.

8 Le caractère de domination qui est dans une certaine mesure inscrit d'emblée dans le concept de bureaucratie avait déjà été souligné par "l'inventeur» du terme, l'économiste français Vincent de Gournay ${ }^{26}$. Robert von Mohl considère la bureaucratie comme un "adversaire » qui " avance avec force ", "crainte et redoutée », et a pris la «domination sur l'État $»^{27}$. Josef Olszewski décrit la manière dont les fonctionnaires se sont vus confier les "affaires de l'État sous forme de monopole ", se sont emparés « du monopole des sciences politiques $»^{28}$ et ont prospéré pour devenir "peu à peu un élément puissant " "qui a progressivement ôté une partie de leur pouvoir antérieur aux gouvernants crédules $»^{29}$. Max Weber pousse encore plus loin cette découverte. Il est clair à ses yeux que les fonctionnaires se sont emparés de toute la domination. Ainsi, comme chez ses prédécesseurs, sa critique de la bureaucratie devient une critique de la fonction publique.

Chez Weber, et contrairement à ses prédécesseurs, on ne trouve cependant pas la polémique corrosive dirigée contre les fonctionnaires au XIX ${ }^{\mathrm{e}}$ siècle. Marx les traite de " jésuites de l'État, théologiens de l'État» dont le credo est "la subordination et l'obéissance passive ", la "chasse aux postes supérieurs " et la «réalisation d'une carrière $»^{30}$. Robert von Mohl constate que toute la population est unie « dans la haine et le mépris à l'égard des bureaucrates " $^{31}$, dont "l'arrogance bornée " et "l'attachement obtus au traditionnel » est une source d'agacement ${ }^{32}$, dont le regard ne va jamais au-delà de leurs "glaciers de dossiers " $33^{33}$ et dont l'activité préférée est "l'écrivaillerie inutile » et «le gaspillage d'encre $»^{34}$. Karl Heinzen, le premier analyste et brillant critique de la bureaucratie, dénonce « l'abus et le caractère pernicieux de la domination des fonctionnaires et des bureaux $»^{35}$. Josef Olszewski méprise "l'obéissance aveugle" des fonctionnaires opportunistes ${ }^{36}$ et se dit persuadé «que l'émancipation à l'égard de la polycratie bureaucratique [...] offre l'unique chemin salutaire contre le terrorisme de la fonction publique $»^{37}$. Max Weber, lui aussi, est tout sauf un apologiste de la fonction publique. Mais comme ce qui lui importe est de "dépasser la grogne stérile, qui remplace la critique positive, à propos du "saint Bureaucratius" $»^{38}$, il se dresse contre cette polémique aussi prisée que vaine pour se tourner au contraire vers une "critique positive». C'est aussi dans ce sens qu'il faut comprendre le sous-titre de «Parlement et gouvernement ». Sa position, comme c'est si souvent le cas, est ambivalente. Face à sa critique des fonctionnaires, on trouve aussi bien la reconnaissance de leur signification historique dans le processus de genèse de l'État que l'admiration de la prestation qu'ils fournissent au profit du fonctionnement de l'État. Il sait que l'histoire du succès de l'État moderne n'est pas en dernier lieu celle de la fonction publique. 

sont des principes structurels bien connus et supérieurs à toute autre structure d'organisation de la domination de l'État : précision, rapidité, calculabilité, hiérarchie, division du travail, efficience. Weber n'est pas le premier à citer ces principes. Hegel considère déjà la bureaucratie comme un appareil de fonctionnaires organisés selon les principes de la «hiérarchie » et des « responsabilités $»^{40}$. Il estime que la division du travail et la centralisation sont nécessaires pour atteindre plus "de simplicité, de rapidité et d'efficacité dans le règlement des affaires qui concernent l'intérêt général de l'État $»^{41}$. Marx, qui reconnaît à Hegel le mérite d'avoir accompli « une description empirique de la bureaucratie ${ }^{42}$ ), souligne aussi bien le caractère nécessairement formaliste de la bureaucratie que la nature forcément bureaucratique de l'État ${ }^{43}$, deux aspects qui jouent un rôle central dans l'analyse de Weber. conception précise de la bureaucratie ${ }^{45}$. Robert von Mohl, qui entreprend tout de même une tentative de définition ${ }^{46}$, ne peut que constater une funeste confusion autour de ce terme déjà partout répandu à son époque: le fait que l'on "parle certes de "bureaucratie" en tout lieu et dans les occasions les plus diverses", mais que chacun entend autre chose sous ce terme ${ }^{47}$. Même Josef Olszewski, qui cite déjà la formation professionnelle, le principe de la classification dans les archives, le devoir de fidélité et l'obligation d'obéissance comme principes de la bureaucratie ${ }^{48}$ - des critères que Weber a indiscutablement pris comme point de repère - ne peut définir la bureaucratie que comme une "corporation organisée de manière hiérarchique " ${ }^{49}$. De manière significative, la bureaucratie n'est pas analysée et discutée avec la bureaucratie dans la Théorie générale de l'État au XIX ${ }^{\mathrm{e}}$ et au début du $\mathrm{XX}^{\mathrm{e}}$ siècles, et on la chercherait aussi en vain chez Jellinek. Weber est certes le premier à tenter d'appréhender plus précisément la relation entre l'État et la bureaucratie, mais comme il ne l'entreprend systématiquement nulle part, il faut commencer par reconstituer cette relation.

On le sait, Max Weber dessine le sombre tableau d'un avenir constitué par la bureaucratisation proprement «totale » de toute la vie sociale et publique, et sa vision apocalyptique ne laisse aucun espoir d'alternative. Les «dominés» ne peuvent «ni se passer de l'appareil bureaucratique de domination, une fois qu'il existe, ni le remplacer", si bien que l'idée d'une sortie de ce système devient "de plus en plus utopique »: « Une fois que la bureaucratisation de l'administration a été intégralement accomplie, on a créé une forme de relations de domination qu'il est pratiquement impossible de briser ${ }^{50}$ », cette prophétie, qui repose sur le diagnostic sociologique du caractère inéluctable et inévitable de la bureaucratie d'État, pourrait être placé sous la devise dantesque: "Lasciate ogni speranza, voi ch'entrate." Max Weber n'est pas le premier à « abandonner toute espérance ». Dès 1845, Karl Heinzen notait, aussi résigné qu'ironique, que la bureaucratie est apparemment «d'origine divine», puisqu'elle est «infaillible et toute puissante $»^{51}$. Pour Marx, la bureaucratie est «un cercle d'où personne ne peut sortir d'un bond ${ }^{52}$. Et John Stuart Mill pronostique l'avènement d'un État où « tout passe par la bureaucratie » et où l'on « ne peut strictement rien faire qui ne convienne pas à celle-ci ${ }^{53}$. Le profond pessimisme de Max Weber se situe donc dans la tradition de certains des analystes les plus perspicaces du XIX ${ }^{e}$ siècle. Comme ceux qui entrent dans l'enfer de Dante, ceux qui se rendent dans le royaume de la bureaucratie décrit par Weber entendent ces paroles en guise de salutation : «Oubliez toute espérance. » 
13 C'est sous le signe de cette résignation qu'il pose sa question empreinte d'angoisse "sur les futures formes politiques d'organisation» de l'État: comment, compte tenu du pouvoir détenu par la "fonction publique d'État », est-il seulement possible de contenir «la monstrueuse surpuissance » de cette catégorie et de la contrôler efficacement ${ }^{54}$ ? En tant que pessimiste, réaliste et anti-utopiste, il montre que l'anarchisme, qu'il considère de toute façon comme puéril, n'offre pas la moindre chance de défaire le nœud gordien de la domination bureaucratique et étatique. Certes, il tombe d'accord avec Bakounine sur l'idée que les dossiers sont la base de la domination bureaucratique, mais juge illusoire l'abolition des uns comme de l'autre. «La pensée naïve du bakouninisme : pouvoir, en détruisant les dossiers, détruire en même temps la base de la [...] "domination", oublie qu'indépendamment des dossiers, il reste le fait que les gens sont réglés pour respecter les normes et les règlements habituels. ${ }^{55}$ » L'indépendance de la bureaucratie lui paraît aussi utopique que la liberté de la domination. Comme toute activité humaine commune est structurée par la domination, une nouvelle domination naîtrait dès le lendemain de la destruction de la précédente.

L'idée que même les révolutions n'offrent aucune chance de détruire la bureaucratie se dégage de deux remarques de Weber. D'une part, il évalue «la simple poursuite du fonctionnement de l'ancienne direction administrative et le prolongement de la validité de ses ordres sous l'autorité des nouveaux détenteurs du pouvoir » pendant la Révolution de Novembre [1918] comme "un exemple excellent» du caractère inéluctable de la bureaucratie ${ }^{56}$. Il montre, d'autre part, que «depuis l'époque du Premier Empire » en France, "l'appareil de domination est resté, pour l'essentiel, le même", et rend "impossible [...] du point de vue purement technique, une "révolution" dans le sens de la création violente d'entités de domination entièrement nouvelles $»^{57}$. À la question de savoir si les révolutions peuvent permettre une sortie radicale de la sphère d'influence de la bureaucratie, il répond catégoriquement par la négative. Les formes d'État vont et viennent, mais la bureaucratie demeure.

Il utilise ainsi un argument qui se situe entièrement dans la lignée d'Alexis de Tocqueville, qui démontre, dans son analyse de l'État français pré- et postrévolutionnaire, que l'appareil administratif de l'Ancien Régime, dont de toute façon les institutions féodales n'existaient plus que nominalement et étaient bureaucratisées depuis très longtemps, ne fut en aucune manière ébranlé par la Révolution française, mais lui survécut sans rupture ${ }^{58}$. Même si l'on ne sait toujours pas aujourd'hui dans quelle mesure Weber connaissait l'œuvre de Tocqueville ${ }^{59}$, il se situe, aussi bien dans ses problématiques que dans ses réponses, plus que n'importe quel autre penseur politique $\mathrm{du} \mathrm{XX}^{\mathrm{e}}$ siècle, dans la tradition du célèbre auteur Français. Aucun des deux ne veut contester de quelque manière que ce soit la signification historique décisive de la Révolution. Le regard de l'un comme de l'autre, sous l'angle de la sociologie de l'État, se porte toutefois derrière la coulisse du théâtre spectaculaire de la Révolution : alors que sur la scène, on décapite et l'on fusille, que les comédiens et les figurants changent, les « intendants ${ }^{60}$ ", eux, restent toujours les mêmes.

En posant son diagnostic, Max Weber peut non seulement s'appuyer sur Tocqueville, mais aussi sur Josef Olszewski, lequel souligne « qu'y compris les modifications les plus radicales de l'ordre social ", sous la Révolution française par exemple, «non seulement n'ont pas éliminé la bureaucratie d'État, mais l'ont au contraire renforcée pour lui faire atteindre l'apogée de son pouvoir $"^{61}$. Il a fallu attendre Heinz O. Ziegler et son étude, autrefois passée inaperçue, pour que soit parachevée une telle interprétation de la 
Révolution dans l'esprit de la sociologie de la domination telle qu'elle est pratiquée chez Tocqueville, Max Weber et Olszewski. Il y démontre que l'administration, « restée relativement intacte après les bouleversements révolutionnaires de la Constitution ", progresse « continuellement vers une centralisation et une bureaucratisation toujours plus fortes", et que la Révolution française n'a fait que prolonger "l'œuvre administrative de centralisation et de rationalisation » entamée par l'Ancien régime ${ }^{62}$.

Ce que dit Max Weber des processus internes aux États vaut tout autant pour expliquer une occupation de l'État par des puissances hostiles: «Un système de fonctionnaires rationnellement ordonné continue à fonctionner sans faille lorsque le pays est occupé par l'ennemi en changeant uniquement les postes les plus élevés »; cela tient selon lui à l'indifférence, à la neutralité et à la formalité de l'appareil, qui «se trouve très facilement disposé à travailler pour quiconque a su s'en rendre maître $»^{63}$. Olszewski fait déjà ce constat lorsque, s'appuyant sur l'exemple "des renversements politiques " du XIXe siècle, il montre que la bureaucratie s'est toujours « soumise à chaque nouveau gouvernement » et qu'elle est toujours en mesure " de s'accommoder instantanément et de s'adapter [...] au nouvel ordre des choses $\|^{64}$. À l'instar d'Olszewski, Weber ne porte pas non plus de jugement de valeur sur cette observation, mais l'énonce comme le constat d'un fait empirique, historique et relevant de la sociologie de l'État. Son diagnostic, qui peut aussi être lu comme un pronostic, a été confirmé non seulement par la théorie de l'État qui lui a immédiatement succédé 65 , mais aussi par l'histoire du $\mathrm{XX}^{\mathrm{e}}$ siècle. La «mise au pas » des fonctionnaires de l'appareil d'État qui s'est déroulée sans heurts dans l'Allemagne nationale-socialiste, a montré que cette «continuité du fonctionnement" peut se faire sans accrocs. En 1933, l'administration n'a pas seulement été « très facilement disposée » à travailler pour les nouveaux détenteurs du pouvoir, mais elle s'est aussi laissé utiliser comme instrument docile de la domination terroriste. Et bien entendu, après 1945, l'appareil des fonctionnaires "a continué à fonctionner sans faille, en changeant uniquement les postes les plus élevés ", d'abord sous la direction des puissances d'occupation, puis dans la République de Bonn.

De la même manière que l'interprétation de l'anarchisme par Weber (qui, au fond, ne le prend absolument pas au sérieux) sa vision du socialisme est placée à l'intérieur d'une problématique relevant de la sociologie de l'État: savoir si le socialisme, compte tenu de la "parenté sociologique» entre le capitalisme et la bureaucratie, offre une possibilité de dépasser cette dernière. Il n'envisage rien de tel, au contraire : alors que dans le capitalisme, "les fonctionnaires étatico-politiques et ceux de l'économie privée " demeurent "comme des corps séparés» et se "contiennent toujours mutuellement ", les deux bureaucraties seraient, sous le régime socialiste, "un corps unique, ayant des intérêts solidaires, et que l'on ne pourrait absolument plus contrôler ${ }^{66}$. Weber conçoit le rapport entre le pouvoir de l'État et celui de l'économie privée comme une sorte de séparation des pouvoirs, dans laquelle il voit la seule garantie que les deux puissances se contiennent mutuellement et ne deviennent pas «totales ». Dans cette perspective, le socialisme représente une élimination de cette séparation des pouvoirs, une disparition aussi lourde de conséquences que de menaces : "La bureaucratie d'État règnerait seule si le capitalisme privé était éliminé ${ }^{67}$. Derrière le socialisme, tel est le diagnostic clairvoyant qu'il porte sur le plan de la sociologie de l'État, ne se dissimule que « le fait objectif de la bureaucratisation universelle ${ }^{68}$.

19 L'histoire a confirmé le jugement que Weber énonçait en 1918, alors que le premier État socialiste avait tout juste six mois. Les États socialistes du XX $\mathrm{XX}^{\mathrm{e}}$ siècle ont donné le jour à 
des bureaucraties qui, dans leur structure autoritaire et totalitaire, dépassent de loin tous les États bureaucratiques de l'histoire, et donnent même à la vision menaçante des «maisons de servitude [Gehäuse] » l'aspect d'un phénomène aimable. Ce n'est pas un hasard si beaucoup d'analystes des bureaucraties d'Europe de l'Est se sont inspirés des positions et des concepts de Max Weber ${ }^{69}$. Personne, sans doute, n'a prédit avec plus de lucidité les conséquences de cette expérience qui a débuté en 1917 et a échoué de manière dramatique en 1989. Weber pronostiquait dès 1894 qu'elle aboutirait obligatoirement à un despotisme et un autoritarisme grotesques de l'État: "Une organisation socialiste attacherait chaque individu à un fil, et ferait converger ces fils entre les mains d'une direction centrale qui orienterait alors chacun dans le sens où elle croirait pouvoir l'utiliser de la manière la plus utile à l'aune de ses connaissances $^{70}$. Paroles prophétiques. Il ne se laisse cependant guider, ni ici, ni ultérieurement, par un affect antisocialiste. Ce qui frappe, dans son analyse, c'est justement le pronostic impartial et clair qu'il porte, dans la perspective de la sociologie de l'État, sur les conséquences du socialisme.

Huit ans plus tôt, déjà, Nietzsche, avec un «coup d'œil sur l'État " tout aussi aigu, diagnostiquait que la «subordination» qui régnait dans «l'État [...] des fonctionnaires " s'intensifierait encore sous le régime socialiste ${ }^{71}$. Celui-ci, écrivait-il, était « le frère cadet et fantasque du despotisme agonisant ", et donc " réactionnaire au sens le plus profond ». Car il désire la puissance étatique à ce degré de plénitude que seul le despotisme a jamais possédé, il surenchérit même sur le passé en visant à l'anéantissement pur et simple de l'individu [...] $\gg^{72}$. L'affinité entre Weber et Nietzsche apparait une nouvelle fois ici. Les deux penseurs de l'État voient dans le socialisme une multiplication exponentielle de la bureaucratie d'État, tous deux le considèrent comme l'origine de la suppression, lourde de conséquences, de la séparation, des pouvoirs, et tous deux pronostiquent les conséquences périlleuses, pour l'individu, d'un appareil centraliste autoritaire. Max Weber porte certes le plus souvent un regard pessimiste sur l'avenir, mais en novembre 1918 il défend une conviction optimiste: "Le bolchevisme est une dictature militaire comme n'importe quelle autre et elle s'effondrera comme toutes les autres ${ }^{73}$. » Cela n'arrivera pourtant que soixante-dix ans plus tard.

\section{Les problématiques de Max Weber}

21 En s'interrogeant, à propos de l'avenir de l'État, sur la possibilité de contrôler la bureaucratie d'État, Weber reprend sur la question de toutes les questions, celle que se sont posée tous les critiques de la bureaucratie, sans exception. C'est sans doute chez Josef Olszewski qu'elle est formulée le plus distinctement : «Comment doit-on se battre avec la bureaucratie ${ }^{74}$ ? Hegel considère que la protection contre l'abus de pouvoir et "l'arbitraire» des fonctionnaires réside d'une part dans le "contrôle exercé d'en haut " par les principes de la " hiérarchie » et « de leurs responsabilités », d'autre part dans le contrôle exercé d'en bas par les compétences « des communes et corporations " 75. Cette solution "fédéraliste ", Heinrich von Treitschke l'a aussi à l'esprit lorsqu'il considère que "seule l'indépendance de provinces fortes" constitue une protection « contre l'autocratie de la bureaucratie $»^{76}$. Selon Robert von Mohl, on peut endiguer l'abus de pouvoir de la bureaucratie premièrement par des propositions pratiques de réformes faites par la «science", deuxièmement par la désétatisation, et 
troisièmement par une professionnalisation des fonctionnaires ${ }^{77}$. Quant à John Stuart Mill, il demande qu'on ampute les compétences de la bureaucratie et qu'on la soumette à la « critique vigilante d'une instance disposant d'une autorité équivalente, extérieure au gouvernement »; il ne révèle cependant pas de quelle " autorité » il doit s'agir ${ }^{78}$.

Alors que toutes ces propositions des penseurs du XIXe siècle se perdent dans des concepts flous, Josef Olszewski ébauche en 1904, pour la première fois, un concept à la formulation achevée, élaboré et d'aspect très « moderne », qui vise une « simplification du droit matériel et formel ", une "rupture avec la manie qui poussait jusqu'ici à compliquer la construction de la machine administrative ", bref : une "simplification de la machine administrative ${ }^{79}$. Mais comme il faudrait, pour assurer le contrôle de la bureaucratie, créer "de nouveau toute une série d'autres appareils et institutions », et qui plus est une "quantité de préceptes et, pour ceux-ci, de commentaires et d'explications ", il est conscient du fait qu'un contrôle de la bureaucratie n'est possible qu'au prix d'une bureaucratie supplémentaire, et ne peut donc qu'en tirer cette conclusion résignée: "Tant que l'État [...] existera, il faudra des fonctions et des fonctionnaires, et ceux-ci exerceront sans doute toujours une influence significative sur le devenir de la société, pour le meilleur et pour le pire ${ }^{80}$.»

23 La thèse de Max Weber sur le caractère inéluctable de la bureaucratie n'est donc pas seulement le résultat de ses études sur la sociologie de la domination. Elle correspond aussi à l'opinion dominante de son époque. Il connaît sans aucun doute les concepts proposés pour contrôler la bureaucratie, mais aussi ses apories: contrôler « d'en haut »? Cela ne mène qu'à un parachèvement de la bureaucratie. Contrôler " d'en bas » par décentralisation ou fédéralisme? Ce ne serait qu'un contrôle exercé par des instances elles aussi bureaucratisées. Contrôler par la science ? Cela reviendrait à un contrôle produit par l'une des institutions les plus bureaucratisées qui soient. La solution qu'envisage Weber ne peut donc plus être celle de l'un de ses prédécesseurs. Il mise sur une institution dont les instruments de contrôle n'étaient pas encore formés au XIX siècle: le parlement, qu'il considère comme "indispensable » en tant qu' « organe du contrôle des fonctionnaires $»^{81}$ et qui permet selon lui, par le biais du droit d'enquête, un " contrôle permanent » de la bureaucratie ${ }^{82}$. Mais le parlement n'est lui aussi qu'une arme émoussée dans le combat pour la bureaucratie, dès lors que «le principal moyen de pouvoir de la fonction publique est de transformer les connaissances liées au service en un savoir secret ", " un moyen de préserver l'administration contre le contrôle $»^{83}$. Il doit en outre reconnaître que les institutions qui définissent le fonctionnement parlementaire, les partis, sont pour leur part soumis à un destin inéluctable : «Ils sont livrés à la bureaucratisation, tout comme l'appareil d'État ${ }^{84}$. » On peut regretter que Weber, le principal analyste de la bureaucratie au $\mathrm{XX}^{\mathrm{e}}$ siècle, ne puisse proposer de solution définitive pour régler ce problème qu'il ressent lui-même comme pesant. Mais son réalisme pessimiste est de toute façon certainement plus honnête que l'activité à vide et le désarroi élaboré des commissions bureaucratiques d'anti-bureaucratisation que l'on voit de nos jours.

On ne trouve donc pas chez Weber de réponse au problème de l'État bureaucratisé, mais sans doute une série de problématiques. Elles sont souvent plus éloquentes et plus importantes que des réponses. "Car Messieurs", dit-il lors du premier Congrès Allemand des Sociologues, «la formulation des problématiques effectives qu'il nous faut élaborer constitue justement la mission scientifique décisive $»^{85}$. Cette mission, il n’a jamais cessé de se l'assigner. Lors du Congrès de l'Association pour la Politique Sociale, 
en 1905, il reconnaît que la "seule question » qui l'intéresse est de savoir ce que deviennent les hommes «du point de vue caractérologique» dans l'État bureaucratique, face à « la sensation autoritaire, au fait d'être règlementé, commandé et restreint », toutes choses qu'apporte " l'État actuel ${ }^{86}$. Quatre ans plus tard, face au "caractère irrésistible du progrès de la mécanisation bureaucratique ", il précise sa problématique : «La question qui nous préoccupe n'est pas : Comment peut-on changer quelque chose à cette évolution? (Car cela, on ne le peut pas), Mais: Qu'est-ce qui en découle ? $\aleph^{87}$ Il donne ici la réponse à la question qu'il avait posée quatre ans plus tôt et d'abord laissée sans réponse: Dans l'État bureaucratique, les gens se mettent à avoir "besoin "d'ordre" et d'ordre uniquement, deviennent nerveux et lâches dès que cet ordre vacille un instant, et sont désemparés lorsqu'ils sont arrachés à leur adaptation exclusive à cet ordre ", et sa " question centrale » est de savoir « ce que nous pouvons opposer à cette machinerie " pour nous préserver de "cette autocratie des idéaux bureaucratiques de l'existence $»^{88}$.

Il est symptomatique que Weber dise que la "réponse à cette question" ne "relève plus aujourd'hui de ce lieu ». Chaque fois qu'il pose la question de la préservation de la liberté et de l'individualité dans l'État bureaucratique, ses propos s'interrompent brutalement, sur la remarque selon laquelle la réponse «ne relève pas aujourd'hui de ce lieu » ou ne « doit pas intéresser cette fois». On le constate aussi lorsqu'il demande comment, "face à la toute puissance de la tendance vers la bureaucratisation, il est seulement encore possible» de sauver "quelque reste que ce soit, dans quelque sens que ce soit, de la liberté de mouvement "individualiste" $"{ }^{89}$. Parce qu'il juge cette progression inéluctable, il est clair à ses yeux qu'il n'y a pas de planche de salut, et il ne connaît donc pas non plus de réponse à cette question. Dans son œuvre, en tout cas, aucun indice ne témoigne de son existence. Il faut partir de l'hypothèse que ses propres découvertes fournissent une réponse obsolète : si la bureaucratie est inéluctable, cela vaut aussi pour ses conséquences, la désindividualisation et la perte de liberté. C'est la raison pour laquelle il écarte la question de savoir « comment on peut changer quelque chose à cette évolution » et se consacre à la seule question qui l'intéresse, celle des conséquences de cette évolution. Cette problématique guide son interprétation de l'État bureaucratique moderne.

Les problématiques que l'on a fait ressortir ici se rapportent sans exception à un phénomène spécifique. Il ne s'agit donc pas ici d'une participation au débat prisé de nos jours qui vise à déterminer le thème de Weber, à la recherche duquel quantité de "spécialistes" de Weber se sont consacrés depuis les années $1980^{\circ 0}$. La controverse portant sur la question de savoir s'il existe une problématique qui régit toute son œuvre a les caractéristiques d'un combat de boxe contre des ombres. Weber n'analyse pas seulement des objets de recherches différents sous des aspects différents mais applique aussi, nous l'avons vu, à chaque objet en soi (comme celui de la bureaucratie) une quantité de problématiques diverses. Celles-ci ont toutefois une direction tout à fait commune : lorsqu'il demande comment on peut préserver l'individualité et la liberté, et limiter le pouvoir de la bureaucratie, il ne peut y avoir aucun doute sur le fait qu'il s'agit de points de vue libéraux. Il se situe dans la tradition de Robert von Mohl, qui considère que «l'autodétermination de l'individu» est menacée dans l'État bureaucratique ${ }^{91}$, dans celle d'Alexis de Tocqueville et de John Stuart Mill, pour lesquels la liberté est menacée dans l'État bureaucratique. Si les digressions historiques de Weber sur la naissance de l'État moderne ont pour objectif de démontrer l'avancée de la démocratie, son analyse de l'État à son époque découle de la question de savoir 
comment la bureaucratie peut être contrôlée et limitée. Stefan Breuer estime même que " toute la théorie politique de Weber » est «faite pour répondre à la question de savoir comment on peut produire suffisamment d'énergie politique pour maintenir la bureaucratie dans le statut d'un simple instrument et endiguer la dynamique répressive qui lui est propre $»^{92}$. Il manque toutefois chez Weber cet optimisme libéral qui vise à fixer des limites à la bureaucratie au moyen de la connaissance scientifique, de la désétatisation ou de la restriction des compétences. Ces conceptions libérales développées par un Robert von Mohl et un John Stuart Mill sont pour lui des rêves pieux.

\section{BIBLIOGRAPHIE}

Albrow, M. (1970) : Bureaucracy, Londres.

Baier, H. (1988) : « "Vater Sozialstaat”. Max Webers Widerspruch zur Wohlfahrtspatronage », dans : Gneuss, C. / Kocka, J. (1988) : Max Weber. Ein Symposion, Munich, p. 47-63.

Bobbio, N. (1987) : The Future of Democracy, Cambridge.

Breuer, S. (1990) : «Rationale Herrschaft. Zu einer Kategorie Max Webers », Politische Vierteljahresschrift, 31, p. 4-32. La traduction française est publiée dans ce numéro de Trivium [NDLR].

Breuer, S. / Treiber, H. / Walther, M. (1982) : «Enstehungsbedingungen des modernen Anstaltstaates. Überlegungen im Anschluss an Max Weber », dans : Breuer, S. / Treiber, H. (éd.) : Entstehung und Strukturwandel des Staates, Opladen, p. 75-153.

Ellwein, Th. (1993) : Der Staat als Zufall und als Notwendigkeit. Die jüngere Verwaltungsentwicklung in Deutschland am Beispiel Ostwestfalen-Lippe, vol. 1, Opladen.

Emge, C. A. (1950/51) : « Bürokratisierung », Kölner Zeitschrift für Soziologie und Sozialpsychologie, 3, p. 179-195.

Forsthoff, E. (1938) : Die Verwaltung als Leistungsträger, Stuttgart / Berlin.

Freund, D. (1974) : « Max Weber und Alexis de Tocqueville », Archiv für Kulturgeschichte, 56, p. $457 \mathrm{sq}$.

Habermas, J. (1981) : Theorie des kommunikativen Handels, 2 vol., Francfort-sur-le-Main. [trad. fr. (1987) : Théorie de l'agir communicationnel, vol. 1 : Rationalité de l'agir et rationalisation de la société, traduit de l'allemand par J.-M. Ferry, Paris : Fayard].

Hattenhauer, H. (1980) : Geschichte des deutschen Beamtentums, Cologne.

Hegel, G. W. F. (1998) : Principes de la philosophie du droit, traduit de l'allemand, présenté et annoté par R. Dérathé, Paris : Librairie philosophique J. Vrin.

Heinzen, K. (1845) : Die Preussische Büreaukratie, Darmstadt.

Hennis, W. (1982) : « Tocquevilles "Neue politische Wissenschaft” ", dans : Stagl, J. (éd.) : Aspekte der Kultursoziologie, Berlin, p. 385-407. 
Hennis, W. (1987) : Max Webers Fragestellung. Studien zur Biographie des Werks, Tübingen.

Hintze, O. (1964a) : « Der Beamtenstand» (1911), dans : Gesammelte Abhandlungen, vol. 2 : Soziologie und Geschichte, éd. par G. Oestreich, Göttingen, p. 66-125.

Hintze, O. (1964b) : « Der Staat als Betrieb und Verfassungsreform » (1927), dans: Gesammelte Abhandlungen, vol. 2 : Soziologie und Geschichte, éd. par G. Oestreich, Göttingen, p. 205-209 [trad. fr. dans : Hintze, O. (1991) : Féodalité, capitalisme et Etat moderne. Essais d'histoire sociale comparée, choisis et présentés par Hinnerk Bruhns, Paris : Editions de la Maison des sciences de l'homme, p. 291-299].

Hirszowicz, M. (1980) : The Bureaucratic Leviathan. A Study in the Sociology of Communism, Oxford.

Käsler, D. (1977) : Revolution und Veralltäglichung. Eine Theorie postrevolutionärer Prozesse, Munich.

Kafka, F. (1976) : Le Château, traduit de l'allemand par A. Vialatte, Paris : Gallimard (La Pléiade).

Kimminich, O. (1975) : «Die Bedeutung des Beamtentums für die Herausbildung des modernen Staates ", dans : Leisner, W. (éd.) : Das Berufsbeamtentum im demokratischen Staat, Berlin, p. 47-70.

Kocka, J. (1981) : « Capitalism and Bureaucracy in German Industrialization before 1914 », The Economic History Review, 34.

Krygier, M. (1979) : « Weber, Lenin and the reality of socialism », dans Kamenka, E. / Krygier, M. (éd.) : Bureaucracy, Londres, p. 61-87.

Lotz, A. (1909) : Geschichte des Deutschen Beamtentums, Berlin.

Luhmann, N. (1968) : « Zweck - Herrschaft - System. Grundbegriffe und Prämissen Max Webers », dans : Mayntz, R. (éd.) : Bürokratische Organisation, Cologne / Berlin, p. 36-55.

Marx, K. (1961) : « Aus der Kritik der Hegelschen Rechtsphilosophie. Kritik des Hegelschen Staatsrechts », dans : Marx, K. / Engels, F. : Werke, vol. I, Berlin, p. 201-336.

Mill, J. S. (1974 [1859]) : Über die Freiheit, Stuttgart.

Mohl, R. von (1962) : « Über Bureaukratie » (1846), dans : id. : Staatsrecht, Völkerrecht und Politik, vol. 2, Graz, p. 99-130.

Nietzsche, F. (1988) : Humain, trop humain, I, traduit de l'allemand par R. Rovini, dans : id. : Cuvres complètes, vol. III, Paris : Gallimard.

Olszewski, J. (1904) : Bureaukratie, Würzburg.

Rehbinder, M. (1987) : « Max Weber und die Rechtswissenschaft », dans : Rehbinder, M. / Tieck, K.-P. (éd.) : Max Weber als Rechtssoziologe, Berlin, p. 127-149.

Ripley, Th. H. (1990) : « Political Legitimacy under Mono-organisational Socialism », dans : id. : The Changing Soviet System, Aldershot, p. 155-182.

Roth, G. (1987) : Politische Herrschaft und persönliche Freiheit, Francfort-sur-le-Main.

Schluchter, W. (1972) : Aspekte bürokratischer Herrschaft. Studien zur Interpretation der fortschreitenden Industriegesellschaft, Munich.

Schmitt, C. (1932) : Legalität und Legitimität, Munich / Leipzig.

Schöllgen, G. (1985) : Max Webers Anliegen, Darmstadt.

Schöllgen, G. (1988) : « Auf der Suche nach dem "Menschentum”. Im Labyrinth der neueren MaxWeber-Forschung ", Historische Zeitschrift, 246, p. 365-384.

Süle, T. (1988) : Preussische Bürokratietradition. Zur Entwicklung von Verwaltung und Beamtenschaft in Deutschland 1871-1918, Göttingen. 
Tocqueville, A. de (1967 [1856]) : L'ancien Régime et la Révolution, Paris : Gallimard (pagination indiquée : édition Folio).

Treitschke, H. von (1903) : Historische und politische Aufsätze, vol. 2, 6e éd., Leipzig.

Wahl, R. (1980) : « Die bürokratischen Kosten des Rechts- und Sozialstaats », Die Verwaltung, 13, p. 273-296.

Weber, M. (2010) : La Bourse, traduit de l'allemand et précédé d'une introduction par Pierre de Larminat. Paris : Editions Allia.

Ziegler, H. O. (1931) : Die moderne Nation. Ein Beitrag zur politischen Soziologie, Tübingen.

Abréviations utilisées pour les œuvres de Max Weber citées en langue allemande:

GARS : Weber, M. (1972) : Gesammelte Aufsätze zur Religionssoziologie, 6éd., Tübingen, vol. 1.

GASS : Weber,M. (1988) : Gesammelte Aufsätze zur Soziologie und Sozialpolitik, Marianne Weber, $2^{\mathrm{e}}$ éd., Tübingen.

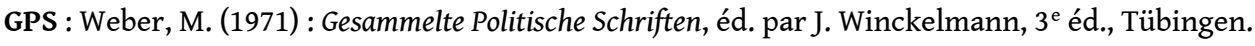

MWG I/15 : Weber, M. (1984) : Zur Politik im Weltkrieg. Schriften und Reden 1914-1918, éd. par W. J. en collaboration avec G; Hübinger, Tübingen.

MWG I/16 : Weber, M. (1988) : Zur Neuordnung Deutschlands. Schriften und Reden 1918-1920, éd. par Wolfgang Mommsen en coll. avec Wolfgang Schwentker, Tübingen 1988.

WuG : Weber,M. (1976) : Wirtschaft und Gesellschaft. Grundriss der verstehenden Soziologie, éd. par J. Winckelmann, $5^{\mathrm{e}}$ éd., Tübingen.

WL : Weber,M. (1973) : Gesammelte Aufsätze zur Wissenschaftslehre, éd. par J. Winckelmann, $4^{\mathrm{e}}$ éd., Tübingen.

\section{Abréviations utilisées pour citer les traductions françaises de Max Weber (NDT) :}

E\&S : Weber, M. (1995) : Économie et Société, traduit de l'allemand par J. Freund, P. Kamnitzer, P. Bertrand, E. de Dampierre, J. Maillard et J. Chavy sous la direction de J. Chavy et d'E. de Dampierre, 2 vol., Paris : Pocket.

ETS : Weber, M. (1965) : Essais sur la théorie de la science, traduit de l'allemand et introduits par J. Freund, Paris : Plon.

OP : Weber, M. (2004) : CEuvres Politiques 1895-1919, traduit de l'allemand par E. Kauffmann, J.P. Mathieu et M.-A. Roy, présentation d'E. Kauffmann, introduction de C. Colliot-Thélène, Paris : Albin Michel.

SR : Weber, M. (1996) : Sociologie des religions, textes réunis et traduits par J.-P. Grossein, Paris : Gallimard.

\section{NOTES}

1. Kafka (1976), p. 710.

2. WuG, p. 559 [L'absence d'une référence à une traduction française d'un texte de Weber signifie qu'une n'existe pas, comme dans le cas présent pour la deuxième partie de Economie et société (N.d.T.)] 
3. WuG, p. 128 [E\&S, p. 298]. Il utilise ici une métaphore qui n'est pas caractéristique de son style. 4. «La confrontation avec cette conception, les va et vient de ses concepts, les tentatives de refonte, d'ajout, de contrôle empirique sont l'objet d'un travail scientifique courant ", comme le note dès les années 1960 Niklas Luhmann, non sans une légère ironie (Luhmann [1968], p. 36). Un certain phénomène de lassitude semble s'être installé aujourd'hui, mais le diagnostic que fait Wolfgang Schluchter d'une « orientation presque obsessionnelle » de beaucoup de sociologues de l'organisation vers Weber (Schluchter [1972], p. 18) reste vraie, puisque la quasi-totalité des travaux dans ce domaine se rattachent à lui - que ce soit pour l'approuver ou pour le récuser. On voit à quel point sa conception a aussi été féconde pour la recherche historique dans la remarquable étude de Tibor Süle (1988), qui, d'une part, utilise le modèle de bureaucratie de Weber comme instrumentaire théorique, et d'autre part le « vérifie » à l'aide d'études historiques empiriques.

5. GASS, p. 499.

6. WuG, p. 560 .

7. WuG, p. 560 sq.

8. Cf. les éléments chez Süle (1988), p. 25 sq. ; cf. aussi Ellwein (1993) ; Wahl (1980) p. 273 sq. ; Bobbio (1987), p. 38.

9. Mohl (1962), p. 111.

10. Olszewski (1904), p. 45.

11. Ibid., p. 274.

12. C'est la thèse intenable de Manfred Rehbinder (1987), p. 138. Sur le rapport entre Weber et l'État social de son époque, cf. Baier (1988), p. 47 sq.

13. Cf. le texte déjà classique d'Ernst Forsthoff (1938), notamment p. 6 sq. Forsthoff n'a pas seulement forgé le concept de "prévoyance » [ "Daseinsvorsorge »] mais a aussi montré - en se rattachant à Weber - que les États, en raison de l'important «besoin de prévoyance » des hommes vivant dans le monde technicisé et urbanisé, connaissent une «croissance extraordinaire de leur pouvoir» (ibid., p. 7 sq.) et deviennent «des entités nécessairement rationalisées » (ibid., p. 9).

14. Süle (1988), p. 26. Il étudie ce processus de manière détaillée et convaincante à l'aide de données statistiques, et étaye notamment ainsi sur un plan empirique et historique, le lien à la règle, la formalisation et la hiérarchisation de la bureaucratie, tels que les décrit Max Weber sous forme idéaltypique (ibid., p. 25 sqq.).

15. WuG, p. 561.

16. GPS, p. 321 sq. [OP, p. 324].

17. GPS, p. 320 [OP, p. 323].

18. WL, p. 477. Sur le rapport entre capitalisme et bureaucratie dans l'œuvre de Max Weber, cf. l'étude de Breuer et al. (1982), p. 134 sq.; sur ce sujet en général, voir aussi Kocka (1981), p. 110 sqq.

19. GARS, I, 3 [trad. fr. : « Avant-Propos », SR].

20. WL, p. 477.

21. Kimminich (1975), p. 47. Approfondissement de la culture générale chez Hattenhauer (1980).

22. Kimminich (1975), p. 50.

23. Albert Lotz ([1909], p. 7) indique par exemple que la naissance de la fonction publique s'est produite « en lien immédiat avec la croissance de l'État moderne ». Otto Hintze (1964a) en arrive à la même conclusion.

24. GARS, I, 3.

25. GPS, p. 320 [OP, p. 323].

26. Vincent de Gournay diagnostique vers 1740 une nouvelle maladie, la " bureaumanie », et une nouvelle forme de domination, la « bureaucratie» : «Nous avons [...] une maladie qui fait bien du ravage; cette maladie s'appelle la bureaumanie. Quelquefois, il en faisait une quatrième ou 
cinquième forme du gouvernement sous le titre de bureaucracie » (cité d'après Emge (1950/51) p. 179).

27. Mohl (1962), p. 104.

28. Olszewski (1904), p. 46.

29. Ibid., p. 44.

30. Marx (1961), p. 248 sq.

31. Mohl (1962), p. 101.

32. Ibid., p. 102.

33. Ibid., p. 103.

34. Ibid., p. 115. Bien qu'il prétende ne faire ici que restituer « de manière neutre » des opinions répandues, on distingue facilement derrière celles-ci ses propres points de vue.

35. Heinzen (1845), p. 13. Il est ravi que ce concept soit au moins un terme étranger : «Le mot Büreaukratie est l'un de ces mots discrédités que nous [...] ne pouvons absolument pas restituer dans notre langue maternelle. C'est un honneur pour notre langue maternelle (et non pour nousmêmes) si nous reprenons même à l'étranger des choses si mauvaises que nous ne savons même pas comment les désigner en allemand. » (ibid.).

36. Olszewski (1904), p. 55.

37. Ibid., p. 50.

38. GPS, p. 354 [OP, p. 359].

39. GPS, p. 321 [OP, p. 324].

40. Hegel (1998), p. 303 (§ 295).

41. Ibid., p. 301 (§ 290 , add.).

42. Marx (1961), p. 247.

43. Ibid., p. 248.

44. On s'en tient à un ensemble de formules sonores et redondantes selon lesquelles la bureaucratie est «le formalisme de l'État », l'« esprit formel de l'État », «l'illusion de l'État ", « l'État imaginaire » ou « le spiritualisme de l'État » (ibid., p. 248 sq.).

45. Sur les approches de théorie de la bureaucratie auxquelles se livrent les penseurs du XIX ${ }^{\mathrm{e}}$ siècle, voir Albrow (1970), p. 18 sq. Il place Weber parmi les théoriciens de l'histoire, avec Michels, Marx et Schmoller (ibid., p. 50 sq.).

46. Robert von Mohl lui-même juge insatisfaisante sa définition, selon laquelle la bureaucratie est « une direction ou une activité quelconque d'un pouvoir gouvernemental quelconque » (Mohl [1962], p. 101).

47. Ibid., p. 100.

48. Olszewski (1904), p. 107 sqq., 95 sqq., 137 sqq. et 147 sqq.

49. Ibid., p. 49.

50. WuG, p. 570 .

51. Heinzen (1845), p. 134.

52. Marx (1961), p. 249.

53. Mill (1974 [1859]), p. 154.

54. GPS, p. 333 [OP, p. 336 sq. ; trad. mod.].

55. WuG, p. 570.

56. WuG, p. 155 [E\&S p. 348].

57. WuG, p. 571. Cela vaudrait la peine d'éclairer un jour de manière plus détaillée le concept wébérien de révolution et de l'analyser du point de vue de l'évolution historique des théories. Sur la disparité des concepts de révolution, cf. Käsler (1977), p. 12 sqq.

58. Tocqueville (1967), notamment p. 45, 47 et 128. Du point de vue "administratif», la Révolution ne marque une césure que dans la mesure où, en éliminant la monarchie absolue et les restes des ordres féodaux, elle créa la condition d'une bureaucratisation forcée. 
59. Dorrit Freund (1974) fait une première tentative d'établir une filiation entre Tocqueville et Weber. Les «indices» biographiques qu'elle présente pour "prouver» que Weber a lu Tocqueville semblent cependant peu crédibles: que Dilthey, qui a fait une remarque sur Tocqueville, ait fréquenté la maison des parents de Weber, ou que Georg Jellinek, qui a mentionné Tocqueville dans sa Théorie générale de l'État, ait été lié d'amitié à Weber, ne signifie rien en soi. - Les indications concises de Wilhelm Hennis (1982), p. 402, en revanche, vont dans la bonne direction.

60. Au théâtre [les « intendants », en Allemagne, sont les directeurs d'un lieu de représentation artistique (N.d.T.)] comme dans l'État: dans l'analyse de Tocqueville, les intendants, fonctionnaires dirigeants de l'administration française, jouent un rôle central.

61. Olszewski (1904), p. 51. Comme Tocqueville, il considère la France comme le pays de la bureaucratisation : "L'action de Napoléon a fait de la France le paradis de la bureaucratie, et elle l'est encore aujourd'hui [...]. Vingt fois, dans cet État, on a décrété et proclamé la liberté et pourtant les formes que l'armée des fonctionnaires d'État y imposent à l'existence dans ce pays, ainsi que l'esclavage des routines, montrent que la liberté s'en tient aux grandes phrases. » (ibid., p. 51 sq.).

62. Ziegler (1931), p. 121, "Les révolutions ne détruisent jamais, mais partout reprennent et intensifient le corps de la centralisation absolutiste, dès lors qu'il correspond bien, d'un point de vue technique, aux besoins de calculabilité de l'activité de l'État et du pilotage uniforme des comportements de masse.» (ibid., p. 86). Ce n'est pas un hasard si son étude a une tonalité centrale wébérienne: elle reconnaît une «obligation» à l'égard de Weber dans son « questionnement fondamental » et dans son approche sociologique (ibid., p. 12.).

63. WuG, p. 570.

64. Olszewski (1904), p. 55 sq.

65. Otto Hintze (1964b), p. 208 - sous l'influence indiscutable de Max Weber - considère comme une «illusion» l'idée que l'on puisse faire l'économie de «l'indispensable machinerie » de la bureaucratie administrative : "Cette bureaucratie représente les piliers et les portants qui ont maintenu l'édifice vacillant de l'État au temps du bouleversement. Son intérêt majeur est de la conserver et de la perfectionner ». [Trad. fr. : Hintze (1991), p. 298 ; trad. mod.] - Carl Schmitt (1932), p. 16, dans la situation virulente de l'année 1932, est encore plus clair: «La "bureaucratie" a souvent à l'égard des différentes formes et des différents types d'États la neutralité de l'instrument purement technique qui [...] peut aussi servir des courants politiques opposés. ». C'est précisément ce qui arriva en Allemagne à peine quelques mois plus tard. Et Carl Schmitt, on le sait, joue un rôle peu glorieux au cours des années suivantes.

66. MWG I/15, p. 615 (GASS, p. 504) [trad. fr. : « Le socialisme », OP, p. 472 sq.].

67. GPS, p. 332 [OP, p. 335 sq.].

68. GPS, p. 330 [OP, p. 333 sq.].

69. Ripley (1990); Roth (1987), notamment p. 66 sqq. Hirszowicz (1980), p. 14 sqq. ; Krygier (1979), p. 70 sqq.

70. GASS, p. 273 sq. [trad. fr. : Weber (2010), p. 51 ; trad. mod.].

71. Nietzsche (1988), p. 265 (§ 441).

72. Ibid., p. 283 (\$ 473).

73. C'est le bilan qu'il dresse dans son discours du 4 novembre 1918 sur le "nouvel ordre politique de l'Allemagne » (MWG I/16, p. 365).

74. Olszewski (1904), p. 273.

75. Hegel (1988), p. 303 (§ 295).

76. Treitschke (1903), p. 385.

77. Mohl (1962), p. 114 et 123.

78. Mill (1974 [1859]), p. 155.

79. Olszewski (1904), p. 278 sq. 
80. Ibid., p. 59 et 280 .

81. GPS, p. 400 [OP, p. 409].

82. GPS, p. 354 [OP, p. 358].

83. GPS, p. 353 [OP, p. 358].

84. GPS, p. 402 [OP, p. 410 ; trad. mod.].

85. GASS, p. 433 sq.

86. GASS, p. 396.

87. GASS, p. 395.

88. GASS, p. 414.

89. GPS, p. 333 [OP, p. 337 ; trad. mod.].

90. Hennis (1987) ; Schöllgen (1985) ; Habermas (1981), vol. 1, p. 295 sq. Voir aussi Schöllgen (1988).

91. Mohl (1962), p. 112.

92. Breuer (1990), p. 20 ; trad. fr. dans le présent numéro de Trivium.

INDEX

Mots-clés : fonctionnaires, bureaucratie, Nietzsche, Kafka, théorie de l'État, polycratie, État social, prévoyance, rendement, socialisme

Schlüsselwörter : Beamte, Bürokratie, Nietzsche, Kafka, Staatslehre, Polykratie, Sozialstaat, Daseinsvorsorge, Leistung, Sozialismus

\section{AUTEURS}

\section{ANDREAS ANTER}

Andreas Anter est professeur de sciences politiques à l'université de Leipzig. Pour plus d'informations, voir la notice suivante. 